\title{
The momentum distribution of the homogeneous electron gas
}

\author{
Markus Holzmann ${ }^{1,2}$, Bernard Bernu ${ }^{2}$, Carlo Pierleoni ${ }^{3}$, Jeremy \\ McMinis $^{4}$, David M. Ceperley ${ }^{4}$, Valerio Olevano ${ }^{5}$, and Luigi Delle Site ${ }^{6}$ \\ ${ }^{1}$ Univ. Grenoble 1/CNRS, LPMMC UMR 5493, Maison des Magistères, 38042 Grenoble, France \\ ${ }^{2}$ LPTMC, UMR 7600 of CNRS, UPMC, Jussieu, Paris, France \\ ${ }^{3}$ Physics Department, University of L'Aquila, Via Vetoio, 67100 L'Aquila, Italy \\ ${ }^{4}$ Dept. of Physics and NCSA, U. of Illinois at Urbana-Champaign, Urbana, IL 61801, USA \\ ${ }^{5}$ Institut Néel, Grenoble, France and \\ ${ }^{6}$ Max-Planck-Institute for Polymer Research, Ackermannweg 10, D 55021 Mainz Germany
}

(Dated: July 31, 2021)

\begin{abstract}
We calculate the off-diagonal density matrix of the homogeneous electron gas at zero temperature using unbiased Reptation Monte Carlo for various densities and extrapolate the momentum distribution, and the kinetic and potential energies to the thermodynamic limit. Our results on the renormalization factor allows us to validate approximate $G_{0} W_{0}$ calculations concerning quasiparticle properties over a broad density region $\left(1 \leq r_{s} \lesssim 10\right)$ and show that near the Fermi surface, vertex corrections and self-consistency aspects almost cancel each other out.
\end{abstract}

PACS numbers: 05.30.Fk, 71.10.Ay, 71.10.Ca, 02.70.Ss

The uniform electron gas (jellium) is one of the most fundamental models for understanding electronic properties in simple metals and semiconductors. Knowledge of its ground state properties, and, in particular, of modifications due to electron correlation are at the heart of all approximate approaches to the many-electron problem in realistic models. Quantum Monte Carlo methods (QMC) 1] have provided the most precise estimates of the correlation energy, electron pair density and structure factor of jellium; basic quantities for constructing and parameterizing the exchange-correlation energy used in density functional theory (DFT) 2 .

Correlations modify the momentum distribution, $n_{k}$, of electrons, and introduce deviations from the ideal Fermi-Dirac step-function. The magnitude of the discontinuity at the Fermi surface $\left(k_{F}\right)$, the renormalization factor $Z$, quantifies the strength of a quasi-particle excitation [3] and plays a fundamental role in Fermi liquid and many-body perturbation theory (GW) for spectral quantities. Whereas the momentum distribution (as well as other spectral information) is inaccessible in current Kohn-Sham DFT formulations, the reduced singleparticle density matrix - the Fourier transform of $n_{k}$ in homogeneous systems - is the basic object in the so-called density-matrix functional theory [4; these theories rely on knowledge of $n_{k}$ of jellium. Inelastic x-ray scattering measurement of the Compton profile of solid sodium [5] have determined $n_{k}$, but experiments for elements with different electronic densities are less conclusive.

In this paper, we calculate $n_{k}$ for the electron gas (jellium) by QMC in the density region $1 \leq r_{s} \leq 10$. Here, $r_{s}=\left(4 \pi n a_{B}^{3} / 3\right)^{-3}$ is the Wigner-Seitz density parameter, $n$ is the density, and $a_{B}=\hbar^{2} / m e^{2}$ is the Bohr radius. In contrast to previous calculations [ 6 , our calculations are based on more precise backflow $(\mathrm{BF})$ wave functions [7, and a careful extrapolation to the thermodynamic

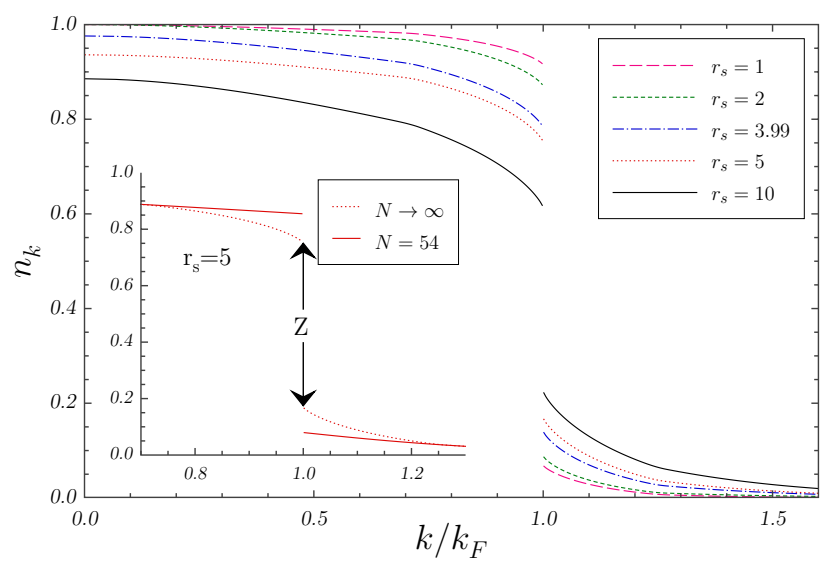

FIG. 1: The momentum distribution $\left(n_{k}\right)$ of the unpolarized electron gas for various densities extrapolated to the thermodynamic limit. The inset shows the extrapolation of $n_{k}$ for $r_{s}=5$ from a system with $N=54$ electrons to the thermodynamic limit, $N \rightarrow \infty$,leading to a significant reduction of the renormalization factor $Z$.

limit [8, 9]. Similar to the worm algorithm in finite temperature path-integral and lattice Monte Carlo [10, 11, we have extended Reptation Monte Carlo (RMC) [12 to include the off-diagonal density matrix in order to obtain an unbiased estimator of the momentum distribution [13, 14]. From our extrapolation scheme, we derive the exact behavior of $n_{k}$ close to the Fermi surface. By comparing the renormalization factor, $Z$, with different approximate GW theories, we can judge the importance of self-consistency and vertex corrections within these approaches. The excellent agreement of our QMC results with $G_{0} W_{0}$ over a broad density region indicate strong cancellations of vertex and self-consistency corrections close to the Fermi surface. 


\begin{tabular}{|c|c|c|c|c|c|}
\hline$r_{s}$ & 1 & 2 & 3.99 & 5 & 10 \\
\hline $\mathrm{E}$ & $1.173(2)$ & $0.0039(1)$ & $-0.1555(1)$ & $-0.1520(1)$ & $-0.1071(1)$ \\
$\mathrm{T}$ & $2.290(3)$ & $0.6024(5)$ & $0.1688(1)$ & $0.1131(1)$ & $0.0349(1)$ \\
$\mathrm{V}$ & $-1.116(1)$ & $-0.5985(1)$ & $-0.3243(1)$ & $-0.2651(1)$ & $-0.1421(1)$ \\
$\mathrm{g}(0)$ & $0.268(3)$ & $0.152(2)$ & $0.057(2)$ & $0.034(1)$ & $0.0036(4)$ \\
\hline$n_{0}$ & 0.999 & 0.998 & 0.97 & 0.93 & 0.88 \\
$n_{2}$ & 0.038 & 0.066 & 0.12 & 0.098 & 0.21 \\
$\bar{n}$ & 0.490 & 0.477 & 0.460 & 0.456 & 0.414 \\
\hline
\end{tabular}

TABLE I: The total $(E)$, potential $(V)$ and kinetic energy $(T)$ per particle in $R y$, and the contact value of the pair correlation function $g(0)$, all extrapolated to the thermodynamic limit from unbiased RMC calculations with backflow (BF) nodes. We further give parameters of the momentum distribution at small $k\left(n_{0}\right.$, and $\left.n_{2}\right) n(k \rightarrow 0)=n_{0}-n_{2}\left(k / k_{F}\right)^{2}$, and at $k_{F}: \bar{n}=\left[n\left(k_{F}^{+}\right)+n\left(k_{F}^{-}\right)\right] / 2$.

Within Variational Monte Carlo (VMC), the ground state wave function is approximated by a trial wave function, $\Psi_{T}(\mathbf{R})$, whereas within projector Monte Carlo methods, e.g. Diffusion Monte Carlo (DMC) or reptation Monte Carlo (RMC), the trial state is improved using $\Psi_{\beta} \propto \exp [-\beta H] \Psi_{T}$; this converges exponentially fast to the true ground state for increasing projection time $\beta$. To circumvent the so-called Fermion sign problem, calculations are done within the fixed-node approximation, introducing small systematic deviations from the exact Fermion ground state [15. Whenever the (approximate) nodes of the system are described by a determinant of single particle orbitals $\phi_{n}(\mathbf{r})$, the (fixed-node) ground state wave function, $\Psi_{N}(\mathbf{R})$, of $N$ particles at positions $\mathbf{R} \equiv\left\{\mathbf{r}_{i}\right\}$, can be written as

$\Psi_{N}(\mathbf{R})=D_{N} \exp \left[-U_{N}\right], \quad D_{N}=\operatorname{det}_{n l} \phi_{n}\left(\mathbf{r}_{l}+\nabla_{l} W_{N}\right)(1)$

where $W_{N}$ and $U_{N}$ are generalized backflow and Jastrow potentials [16 respectively.

From an approximate ground state wavefunction, $\Psi_{N}(\mathbf{R})$, we obtain the reduced single particle density matrix [17]

$$
f_{N}(\mathbf{r})=\langle F(\mathbf{R} ; \mathbf{r})\rangle_{N}, \quad F=\frac{1}{N} \sum_{i} \frac{\Psi_{N}\left(\mathbf{R}: \mathbf{r}_{i}+\mathbf{r}\right)}{\Psi_{N}(\mathbf{R})}
$$

where $\mathbf{R}: \mathbf{r}_{i}+\mathbf{r}$ indicates that the position of particle $i$ is displaced by $\mathbf{r}$, and $\langle\ldots\rangle_{N} \equiv \int d \mathbf{R} \ldots\left|\Psi_{N}\right|^{2} / Q$ with $Q \equiv$ $\int d \mathbf{R}\left|\Psi_{N}\right|^{2}$ playing the role of a partition function. The Fourier transform of $f_{N}(\mathbf{r})$ directly yields the momentum distribution, $n_{\mathbf{k}}^{N}$, of the electrons per spin

$$
n_{\mathbf{k}}^{N}=\frac{1}{2 V} \int d \mathbf{r} e^{-i \mathbf{k} \cdot \mathbf{r}} f_{N}(\mathbf{r})
$$

where $V$ is the volume.

The large variance of the estimator of the off-diagonal density matrix, Eq. (2), makes precise calculations very

\begin{tabular}{|c|c|c|c|c|c|}
\hline$r_{s}$ & 1 & 2 & 3.99 & 5 & 10 \\
\hline BF-RMC & $0.84(2)$ & $0.77(1)$ & $0.64(1)$ & $0.58(1)$ & $0.40(1)$ \\
\hline SJ-VMC & $0.894(9)$ & $0.82(1)$ & $0.69(1)$ & $0.61(2)$ & $0.45(1)$ \\
BF-VMC & $0.86(1)$ & $0.78(1)$ & $0.65(1)$ & $0.59(1)$ & $0.41(1)$ \\
\hline \hline$G_{0} W_{0}[25]$ & 0.859 & 0.768 & $0.646^{*}$ & 0.602 & 0.45 \\
$G W_{0}[26]$ & & 0.804 & $0.702^{*}$ & & \\
$G W[27]$ & & 0.846 & $0.793^{*}$ & & \\
Lam [28] & 0.896 & 0.814 & $0.615^{*}$ & 0.472 & \\
RPA[28] & 0.843 & 0.700 & $0.442^{*}$ & 0.323 & \\
SJ-DMC [6] & 0.952 & 0.889 & & 0.725 & 0.593 \\
\hline
\end{tabular}

TABLE II: Renormalization factor, $Z$, extrapolated to the thermodynamic limit from unbiased RMC calculations with backflow nodes (BF-RMC), together with SJ-VMC, and BFVMC results, compared with perturbative results from literature (literature values* are at $r_{s}=4$ instead of $r_{s}=3.99$ ). Previous SJ-DMC results [6] used mixed estimators without thermodynamic limit extrapolation.

time-consuming. To reduce the variance for homogeneous systems with plane wave orbitals: $\phi_{n}(\mathbf{r}) \propto e^{i \mathbf{k}_{n} \cdot \mathbf{r}}$, we separate the ideal gas density matrix, $f_{i d}(\mathbf{r})=$ $\sum_{n} \phi_{n}^{*}(\mathbf{r}) \phi_{n}(0) / \sum_{n}\left|\phi_{n}(0)\right|^{2}$, based on the estimator

$$
F_{i d}(\mathbf{R} ; \mathbf{r})=\frac{1}{N} \sum_{i} \frac{D_{N}\left(\mathbf{R}: \mathbf{r}_{i}+\mathbf{r} ; W_{N}(\mathbf{R})\right)}{D_{N}\left(\mathbf{R} ; W_{N}(\mathbf{R})\right)}
$$

where the determinants on the r.h.s. of Eq. (4) are evaluated using the backflow coordinates, $W_{N}(\mathbf{R})$, of the diagonal configuration $\mathbf{R}$ with un-displaced particle coordinates. Expanding it around $\mathbf{r}=0$, we can explicitly verify that $f_{i d}(\mathbf{r})=\left\langle F_{i d}(\mathbf{R} ; \mathbf{r})\right\rangle_{N}$, so that the $F-F_{i d}$ is a reduced variance estimator [18] of the difference: $f_{N}-f_{i d}$.

There is a problem with projecting methods to calculate properties other than the energy. Forward walking or reweighting methods based on using $\Psi_{\beta}$ in Eq. (2), become very inefficient for long projection time, since the variance increases exponentially with $\beta$. To avoid this problem, mixed estimators, based on $\Psi_{\beta} \Psi_{0}$, are frequently used but they can introduce a systematic bias. Unbiased estimators for the pair correlation function, potential and kinetic energy have been obtained within RMC [12. Based on a generalized partition function, $\mathcal{Q}$, we extend RMC to include sampling of off-diagonal matrix elements [10]

$$
\begin{aligned}
\mathcal{Q} & =\int d \mathbf{R}\left|\Psi_{\beta / 2}(\mathbf{R})\right|^{2} \\
& +\frac{s}{N} \sum_{i} \int \frac{d \mathbf{r}}{V} \int_{0}^{\beta} \frac{d \tau}{\beta} \int d \mathbf{R}\left|\Psi_{\beta-\tau}(\mathbf{R}) \Psi_{\tau}\left(\mathbf{R}: \mathbf{r}_{i}+\mathbf{r}\right)\right|
\end{aligned}
$$

where $s$ is a parameter used to optimize the efficiency ( $s=0$ corresponds to the usual diagonal RMC [12]). Similar to the worm-algorithm used in continuous Pathintegral calculations [11], our calculations include moves 
which "open" (or "close") a path from diagonal space $\mathbf{R}$ to off-diagonal space $\left(\mathbf{R}, \mathbf{r}_{i}+\mathbf{r}\right)$. Such moves are included at $\tau=0$ and "propagated" by reptation moves [12, 19] to the interior of the path $(\tau>0)$. In contrast to previous calculations using so-called mixed estimators [6], this generalization gives an unbiased estimator of the off-diagonal density matrix, $f_{N}(\mathbf{r})$, and the momentum distribution, $n_{k}^{N}$. Reduction of the variance based on the considerations above, Eq. (4), is still possible, but less effective.

Quantum Monte Carlo results are obtained for typically $N \lesssim 10^{3}$ electrons. The extrapolation to the thermodynamic limit introduces important quantitative and qualitative changes of the momentum distribution around the Fermi surface, $k_{F}$ [9]. For a homogeneous periodic system, the orbitals are plane waves: $\phi_{n}(\mathbf{r})=$ $\exp \left[i\left(\mathbf{k}_{n}+\vec{\theta}\right) \cdot \mathbf{r}\right]$, in the Slater determinant of Eq. (1), where $\mathbf{k}_{j} \in \mathbf{G}_{N} \equiv\left\{\left(n_{1}, n_{2}, n_{3}\right) 2 \pi V^{-1 / 3}\right\}$ with integer $n_{i}$, and $\vec{\theta}$ can be chosen to introduce twisted boundary conditions [8, 20. For a normal Fermi liquid, we further have $\left|\mathbf{k}_{j}+\vec{\theta}\right| \leq k_{F}$, and the generalized backflow and Jastrow potential $W_{N}$ and $U_{N}$ can be written exclusively in terms of collective coordinates $\rho_{\mathbf{k}}=\sum_{n} e^{i \mathbf{k} \cdot \mathbf{r}_{n}}$ and their gradients [7, 16]. Using the wavefunction "potentials", $W_{N}$ and $U_{N}$, expressed as continuous functions in terms of the collective coordinates, the relation between the wave function in the limit $N \rightarrow \infty$ to a finite system is well defined, as it just amounts to evaluations on a denser grid in k-space [8, 9].

Let us first discuss the finite size scaling for a SlaterJastrow (SJ) wave function: a determinant with $W_{N} \equiv 0$, together with a two-body Jastrow correlation, $U_{N}=$ $\sum_{k} u_{k} \rho_{k} \rho_{-k} / 2 V$. We further assume that the function $u_{k}$ is analytically given. In our SJ-VMC calculations, we use the Gaskell form $2 n u_{k}^{S J} \equiv-S_{0}^{-1}(k)+$ $\left[S_{0}^{-2}(k)+2 n v_{k} / \varepsilon_{k}\right]^{1 / 2}$ where $S_{0}(k)$ is the ideal gas structure factor, $v_{k}=4 \pi e^{2} / k^{2}$, and $\varepsilon_{k}=\hbar^{2} k^{2} / 2 m$ [21, 22]. Neglecting mode-coupling between single particle modes in $D_{N}$ and collective modes described by $U_{N}$, the single particle density matrix, Eq. (2), can be approximated as

$$
f_{N}(\mathbf{r}) \approx f_{c}(\mathbf{r}) \equiv\left\langle\frac{D_{N}^{\prime}}{D_{N}}\right\rangle_{N}\left\langle e^{-\left(U_{N}^{\prime}-U_{N}\right)}\right\rangle_{N}
$$

where the prime indicates the off-diagonal configuration, e.g. $D_{N}^{\prime} \equiv D_{N}\left(\mathbf{R}: \mathbf{r}_{1}+\mathbf{r}\right)$. Within the cumulant and rotating wave approximation, we then obtain an explicit expression,

$$
\begin{aligned}
f_{c}(\mathbf{r}) & \simeq f_{i d}(r) \exp \left[-x_{N}(r)\right] \\
x_{N}(r) & =\frac{1}{V} \sum_{|\mathbf{k}| \leq k_{c}}\left[u_{k}\left(S_{k}-1\right)+n u_{k}^{2} S_{k}\right]\left[e^{i \mathbf{k} \cdot \mathbf{r}}-1\right]
\end{aligned}
$$

where $S_{k}=\left\langle\rho_{\mathbf{k}} \rho_{-\mathbf{k}}\right\rangle_{N} / N$ is the structure factor, $f_{i d}(r)=$ $2 \sum_{k \leq k_{F}} e^{i \mathbf{k} \cdot \mathbf{r}} / N$ is the single particle density matrix of the corresponding ideal gas, and we have neglected contributions of short wave length modes, $k_{c} \approx 0.48 r_{s}^{1 / 2} k_{F}$ [23. Further, we can use $S_{k} \approx\left[2 n u_{k}+1 /\left.S_{0}(k)\right|^{-1}\right.$ to express $S_{k}$ in terms of $u_{k}$ and $S_{0}(k)$, which is based on assuming gaussian statistics for $\rho_{k}$, so than Eq. (7) gives an explicit expression for $f_{N}(\mathbf{r}) \approx f_{c}(\mathbf{r})$ in terms of a given Jastrow factor. Whereas the resulting model, Eq. (7), depends weakly on $k_{c}$, so that $f_{N}(r)$ and $n_{k}$ are only qualitatively described, the size-extrapolation is quantitatively correct, as it is dominated by the Jastrow singularity $u_{k} \rightarrow\left(v_{k} / 2 n \varepsilon_{k}\right)^{1 / 2}$ and $S_{k} \rightarrow\left(2 n v_{k} / \varepsilon_{k}\right)^{-1 / 2}$ for $k \rightarrow 0$ stemming from the plasmon contributions.

Since we expect that mode-coupling is negligible in the long wave length limit, the cumulant expression, Eq. (7), can be used to determine the size corrections of QMC calculations of the finite system

$$
f_{\infty}(\mathbf{r})=\frac{2}{n} \int \frac{d^{3} k}{(2 \pi)^{3}} n_{k}^{N} e^{i \mathbf{k} \cdot \mathbf{r}} e^{-\left(x_{\infty}(r)-x_{N}(r)\right)}
$$

Here $n_{k}^{N}$ is the momentum distribution of the $N$ electron system, defined for all values of $\mathbf{k}$ in a grand canonical ensemble using twisted boundary conditions [8]. From the Fourier transform of $f_{\infty}(\mathbf{r})$, Eq. (9), we obtain the extrapolated momentum distribution, $n_{k}^{\infty}$. A related linearized expression has been used to extrapolate the momentum distribution of the two-dimensional electron gas in Ref. [9].

Following the analysis of Ref. 9, leading order corrections to the renormalization factor, $Z_{N}=n_{k_{F}-}^{N}-n_{k_{F}+}^{N}$, are given by

$$
\begin{aligned}
Z_{\infty} & \simeq Z_{N} \exp \left[-\Delta_{N}\right] \\
\Delta_{N} & =\int_{-\pi / L}^{\pi / L} \frac{d^{3} q}{(2 \pi)^{3}} \frac{u_{q}}{2}\left[1+\mathcal{O}\left(\left[2 n u_{q} S_{0}(q)\right]^{-1}\right)\right] \\
& =c\left(\frac{3}{4 \pi}\right)^{1 / 3}\left(\frac{r_{s}}{3}\right)^{1 / 2} N^{-1 / 3}+\mathcal{O}\left(N^{-2 / 3}\right)
\end{aligned}
$$

where $c \simeq 1.221$ is a numerical factor to account for the cubic integration volume [24]. Whereas the asymptotic region is only reached for large systems with $N^{1 / 3} r_{s}^{1 / 2} \gg$ 1, the extrapolation based on the full expression, Eq. (9), includes corrections beyond the leading order term. Analyzing Eq. 99 around $k_{F}$, we obtain the exact leading order behavior with an infinite slope at $k_{F}$

$$
\begin{aligned}
n(k & \left.\rightarrow k_{F}^{ \pm}\right) \simeq n\left(k_{F}^{ \pm}\right) \\
& +\frac{Z_{\infty}}{2 \pi}\left(\frac{9 \pi}{4}\right)^{1 / 3} \sqrt{\frac{r_{s}}{3}}\left[\frac{k}{k_{F}}-1\right] \log \left|\frac{k}{k_{F}}-1\right| .
\end{aligned}
$$

Size extrapolation, discussed above, requires the knowledge of the structure factor, $S_{k}$, and the Jastrow potential, $u_{k}$, in Eq. (8). The QMC calculation of the $N$-particle system allows us only to determine them on a finite grid in $\mathbf{k}$ space, but the analytic continuation to 
the dense grid can be done by interpolation from their known behavior at small k 8 . Whereas $S_{k}$ can be calculated directly, $u_{k}=u_{k}^{S J}$ is only known explicitly for VMC calculations using a Slater-Jastrow trial function. In general, imaginary time projection and backflow introduce an effective Jastrow potential, $u_{k}$, different from the explicitly given form of the underlying trial wavefunction. Expecting small changes at long wave length, $u_{k}=u_{k}^{S J}+\delta u_{k}$, we obtain the modifications $\delta u_{k}$ from from changes in the structure factor $\delta S_{k}=S_{k}-S_{k}^{S J}$ by linear response. For our purpose, mode coupling can be neglected, as well as deviations from gaussian statistics, so that $\delta S_{k} / \delta u_{k^{\prime}} \simeq-2 n S_{k}^{-2} \delta_{\mathbf{k}, \mathbf{k}^{\prime}}$ for $k \rightarrow 0$. Therefore, the effective Jastrow factor of wave functions including backflow and projection can be determined from the structure factor.

Using SJ-VMC calculations with $u_{k}^{S J}$ for $N=54$ to $N=1024$ electrons, we have checked that size extrapolations based on Eq. (9) with $N=54$ are reliable. Thus, the more expensive backflow VMC and RMC calculations based on the analytical wave functions in Ref. [7] are only done with that size. Extrapolated results on the total energy $E$, unbiased estimators from reptation for the potential $(V)$ and kinetic energies $(T)$, and the contact value of the pair correlation function, $g(0)$, are given in table If. The momentum distribution is shown in Fig. 1. The values for the renormalization factor, $Z$, together with different perturbative results from the literature are given in table II Table II also contains the values of the momentum distribution at the origin, $n_{0}$, the negative slope at the origin, $n_{2}$, and $\bar{n}=\left(n_{k_{F}^{-}}+n_{k_{F}^{+}}\right) / 2$. These values can be used to parameterize the momentum distribution along the lines given in Ref. [30, together with $Z$, the exact large $k$ asymptotics [29], $n(k \rightarrow \infty)=(9 / 2) r_{s}^{2} g(0) / k^{8}$, and the exact behavior close to the Fermi surface, Eq. (11). Whereas the mixed estimator usually employed in DMC calculations, introduces a small bias in the momentum distribution, size extrapolation introduces large systematic modifications which limit the precision of the calculations. Previous DMC results [6], using mixed estimators and SJ nodes, suffer from these strong finite size effects and overestimate $Z$ by a large amount.

In summary, we have calcuated the momentum distribution using a new unbiased and much more accurate Monte Carlo method, and extrapolated the results to the thermodynamic limit. In particular, our data allows a quantitative comparison of the renormalization factor, $Z$, with approximate calculations (see table III). The excellent agreement of our results with $G_{0} W_{0}$ [25, 31, 32. over the whole metallic density region $r_{s} \lesssim 5$, strongly indicates that vertex corrections and self-consistency issues - neither is included in $G_{0} W_{0}$ - are canceling each other, at least close to the Fermi surface.

Computer time at CNRS-IDRIS is acknowledged, Projet IDRIS 061801. CP is supported by IIT under the
SEED project grant n 259 SIMBEDD.

[1] D. M. Ceperley and B. J. Alder, Phys. Rev. Lett. 45, 566 (1980).

[2] P. Hohenberg and W. Kohn, Phys. Rev. 136, B864 (1964); W. Kohn and L. J. Sham, Phys. Rev. 140, A1133 (1965).

[3] P. Nozières, Theory of interacting Fermi systems, W.A. Benjamin, Inc., New York, (1964).

[4] T. L. Gilbert, Phys. Rev. B 12, 2111 (1975); P. O. Lodwin, Phys. Rev. 97, 1474 (1955).

[5] S. Huotari, J. A. Soininen, T. Pylkknen, K. Hmlinen, A. Issolah, A. Titov, J. McMinis, J. Kim, K. Esler, D. M. Ceperley, M. Holzmann, and V. Olevano, Phys. Rev. Lett. 105, 086403 (2010).

[6] G. Ortiz and P. Ballone, Phys. Rev. B 50, 1391 (1994); ibid. Phys. Rev. B 56, 9970 (1997).

[7] M. Holzmann, D. M. Ceperley, C. Pierleoni, and K. Esler, Phys. Rev. E 68, 046707 (2003).

[8] S. Chiesa, D. M. Ceperley, R. M. Martin, and M. Holzmann, Phys. Rev. Lett. 97, 076404 (2006).

[9] M. Holzmann, B. Bernu, V. Olevano, R. M. Martin, and D. M. Ceperley, Phys. Rev. B 79, 041308 (2009).

[10] G. Carleo, F. Becca, S. Moroni, and S. Baroni, Phys. Rev. E 82, 046710 (2010).

[11] M. Boninsegni, N. Prokofev, and B. Svistunov, Phys. Rev. Lett. 96, 070601 (2006).

[12] S. Baroni and S. Moroni, Phys. Rev. Lett. 82, 4745 (1999).

[13] B. Militzer and E. L. Pollock , Phys. Rev. Lett. 89, 280401 (2002); B. Militzer, E. L. Pollock, and D. M. Ceperley, arXiv:cond-mat/0310401(2003).

[14] S. Moroni and M. Boninsegni, J. Low Temp. Phys. 136, 129 (2004).

[15] J. Kolorenc and L. Mitas, Rep. Prog. Phys. 74, 026502 (2011).

[16] M. Holzmann, B. Bernu, and D. M. Ceperley, Phys. Rev. B 74, 104510 (2006).

[17] W. L. McMillan, Phys. Rev. 138, A442 (1965).

[18] This variance reduction can be generalized to periodic systems with Bloch orbitals, $\phi_{n \mathbf{k}}(\mathbf{r}) \propto e^{i \mathbf{k} \cdot \mathbf{r}} \varphi_{n}(\mathbf{r})$, displacing only the phase in the determinant, $\phi_{n \mathbf{k}}\left(\mathbf{r}_{i}\right) \rightarrow$ $e^{i \mathbf{k r}} \phi_{n \mathbf{k}}\left(\mathbf{r}_{i}\right)$ when $\mathbf{r}_{i} \rightarrow \mathbf{r}_{i}+\mathbf{r}$.

[19] C. Pierleoni and D. M. Ceperley, ChemPhysChem. 6, 1 (2005).

[20] C. Lin, F. H. Zong, and D. M. Ceperley, Phys. Rev. E 64, 016702 (2001).

[21] T. Gaskell, Proc. Phys. Soc. 77, 1182 (1961); T. Gaskell, Proc. Phys. Soc. 80, 1091 (1962).

[22] D.M. Ceperley, Phys. Rev. B 18, 3126 (1978).

[23] D. Bohm and D. Pines, Phys. Rev. 92, 609 (1953); D. Pines, Elementary Excitations in Solids (Perseus Books, Reading, MA, 1969).

[24] M. Holzmann, B. Bernu, and D.M. Ceperley (to be published).

[25] L. Hedin, Phys. Rev. 139, A796 (1965).

[26] U. von Barth and B. Holm, Phys. Rev. B 54, 8411 (1996); 55, 10 120(E) (1997).

[27] B. Holm and U. von Barth, Phys. Rev. B 57, 2108 (1998).

[28] J. Lam, Phys. Rev. B 3, 3243 (1971). 
[29] J.C. Kimball, J. Phys. A 8, 1513 (1975); H. Yasuhara and Y. Kawazoe, Physica A 85, 416 (1976).

[30] P. Ziesche, Phys. Stat. Sol. (b) 232, No. 2, 231 (2002); P. Gori-Giorgi and P. Ziesche, Phys. Rev. B 66, 235116 (2002).
[31] G. E. Simion and G. F. Giuliani, Phys. Rev. B 77, 035131 (2008).

[32] T.M. Rice, Annals of Physics 31, 100 (1965). 